\title{
Role of Magnetic Resonance Urography in Diagnosis of Pediatric Obstructive Uropathy: A Surgeon Perspective
}

${ }^{1}$ Sameh AbdelHay AbdelHamid, ${ }^{2}$ Khaled AbouelFotouh Ahmed ${ }^{3}$ Mohamed Soliman EIDebeiky, ${ }^{3}$ Ahmed Bassiouny Arafa ${ }^{3}$ Mohamed AbdelSattar Mohamed, Mostafa Mohamed Abdullah ElGhandour

${ }^{1}$ Department of Pediatric Surgery and ${ }^{2}$ Department of Radiology Faculty of Medicine, Ain Shams University, Cairo, Egypt

Corresponding author: Mostafa Mohamed Abdullah ElGhandour, Mobile: 01003703623, E-mail: ghandour31@hotmail.com

\begin{abstract}
Background: Obstructive uropathy is a group of different pathologies. Congenital obstructive uropathy represents a major cause of renal failure in infants and children. Urinary tract obstruction is defined as any condition that impairs urinary drainage from the pelvicalyceal system and leads to increased pressure and reduced urine flow rate.

Objective: The aim of this study was to assess the role of combined static and dynamic Magnetic Resonance Urography in the management of pediatric obstructive uropathy and to compare it with our basic investigations.

Patients and Methods: This study was conducted on the pediatric patients presented to Pediatric Surgery Department in cooperation with Radiology Department, Ain Shams University Hospitals, presented with upper urinary tract dilatation detected by ultrasonograghy.

Results: There was a high agreement between MRU and our standard imaging data as regard detection of urinary tract obstruction giving the MRU no superiority to the basic imaging in this field. Combined MRU showed the highest accuracy among other investigations in identification of the level of obstruction. Dynamic MRU and renal scintigraphy in our study yielded similar results for identification of split renal function.

Conclusion: This study provides evidence that combined static and dynamic MRU is a promising technique that allows anatomical and functional evaluation of obstructed kidneys to be used as a single modality for the comprehensive evaluation of urinary tract obstruction.
\end{abstract}

Keywords: MRU, obstructive uropathy

\section{INTRODUCTION}

Obstructive uropathy in the pediatric age group is a group of different pathologies. The differential diagnosis of urinary tract dilatation includes ureteropelvic junction obstruction, ureterovesical junction obstruction, ectopic ureteral insertion with and without duplex system, posterior urethral valves, urethral atresia, and duplicating collecting system with and without ureterocele. Congenital obstructive uropathy represents a major cause of renal failure in infants and children. Together with renal dysplasia, it leads to almost half of all cases of chronic kidney diseases in children ${ }^{(l)}$.

Urinary tract obstruction is any condition that impairs urinary drainage from the pelvicalyceal system and leads to increased pressure and decreased urine flow rate ${ }^{(2)}$. Clinically, obstruction is usually considered when hydronephrosis is detected by ultrasound. But not every patient with hydronephrosis has an obstruction, and the majority of cases of antenatal hydronephrosis will improve without surgical intervention or renal damage ${ }^{(3)}$.

The pediatric surgeon asks two questions: is there a need to operate, and if so, when? Unfortunately, answering these questions is not straightforward. More than half of hydronephrotic kidneys in infants are not obstructed and will show resolution or will markedly improve without deterioration of renal function. Furthermore, hydronephrosis may prevent renal damage by keeping the pressure inside the renal pelvis low: dilatation does not always indicate obstruction ${ }^{(4)}$.

The well-established methods for investigation still have some defects. 
Ultrasound does not provide a complete assessment and demonstration of the entire urinary tract (e.g. ureter). It cannot evaluate urinary drainage or the grade of obstruction and suffers from significant observer dependency. Renal Scintigraphy is an ionizing method that allows a functional evaluation but offers only poor anatomical resolution and has limitations in poorly or non-functioning kidneys. In upper urinary tract dilatation, the most important imaging task is the differentiation between obstructive and non-obstructive dilatation to allow a decision on therapy (to operate or to treat conservatively). In some conditions, this goal is only partially achievable with the present imaging modalities ${ }^{(5)}$.

The start using magnetic resonance urography has facilitated the assessment of both the function and morphology of the upper urinary tract without radiation. Magnetic resonance urography has been described as accurate in infants and children allowing an evaluation of parenchymal perfusion and glomerular filtration as well as visualization of renal excretory function and urine drainage. This makes MRU appear ideal for a comprehensive evaluation of the entire upper urinary tract, probably replacing other ionizing imaging techniques, however, at the cost of the need for sedation or anesthesia in infants and young children ${ }^{(6)}$.

The aim of this study was to assess the role of combined (static and dynamic) MRU in decision making in the management of pediatric upper urinary tract dilatation in comparison to our basic investigations.

\section{Patients and Methods}

This prospective study included all the pediatric patients with upper urinary tract dilatation detected by ultrasonograghy, attending at Pediatric Surgery Department in cooperation with Radiology Department, Ain Shams University Hospitals. Approval of the ethical committee and a written informed consent from all the children's caregivers were obtained. This study was conducted over a period of 2 years duration between August 2016 till July 2018.
Inclusion criteria: Pediatric patients that have upper urinary tract dilatation detected by ultrasound.

Exclusion criteria: Patients with impaired kidney functions. Patients with ferromagnetic implants were excluded from the study.

Patients who had VUR performed their MRU study but urinary catheter was inserted from the start.

Children with dilated UT on US imaging were investigated with combined static and dynamic MRU in addition to the standard imaging protocol.

Patients were studied through the following regimen: Clinical history: History of febrile attacks, intermittent abdominal colics, recurrent episodes of vomiting, presence of abdominal distention and delayed voiding. Kidney function test: serum creatinine level. Renal ultrasonography: to assess the degree of renal pelvic dilatation, the parenchymal thickness, the ureteric diameter and bladder wall thickening. Voiding cystourethrogram: to detect vesicoureteric reflux, bladder wall irregularity, and dilated posterior urethra. Renal scintigraphy: assessment of split renal function, half-life (T1/2) for isotope excretion and presence of renal scarring. IVP: to assess the urinary system anatomy and excretion

All studies for every case were performed within a period of $6 \mathrm{~m}$ without any surgical intervention in between.

\section{Data Management and Analysis:}

The collected data was revised, coded, tabulated and introduced to a PC using Statistical package for Social Science (SPSS 20). Data was presented and suitable analysis was done according to the type of data obtained for each parameter.

Descriptive statistics: Mean, Standard deviation $( \pm \mathrm{SD})$ and range for numerical data. Frequency and percentage of non-numerical data.

Analytical statistics: Kappa statistics to compute the measure of agreement between two investigational methods Kappa's over 0.75 is excellent, 0.40 
to 0.75 is fair to good, and below 0.40 is poor. Lin's concordance Coefficient ( $\rho$ c) is used to assess the degree of agreement between two continuous variables, such as chemical or microbiological concentrations. It calculates the value of Lin's concordance correlation coefficient.

\section{RESULTS}

\section{Classification of patient's diagnosis:}

The study included 9 patients as shown in table 1

As one of the cases has single kidney (the duplex kidney case), we divided our results according to the renal units considering each kidney as a single unit and the duplex kidney is 2 units(upper and lower moiety) with total number of units being 18 units.

Table (1): Classification of patients diagnosis.

\begin{tabular}{||l|l|c|c|}
\hline \multicolumn{1}{|l|}{} & $\mathbf{N}$ & $\mathbf{\%}$ \\
\hline \multirow{5}{*}{ Diagnosis } & $\begin{array}{l}\text { Persistent } \\
\text { pelvicalyceal } \\
\text { dilatation }\end{array}$ & 1 & $11.1 \%$ \\
\cline { 2 - 4 } & $\begin{array}{l}\text { Follow up after } \\
\text { pyeloplasty }\end{array}$ & 1 & $11.1 \%$ \\
\cline { 2 - 4 } & horse shoe kidney & 1 & $11.1 \%$ \\
\cline { 2 - 4 } & Left duplex kidney & 1 & $11.1 \%$ \\
\cline { 2 - 4 } & Left puj obstruction & 2 & $22.2 \%$ \\
\cline { 2 - 4 } & Left ureteric stricture & 1 & $11.1 \%$ \\
\cline { 2 - 4 } & neurogenic bladder & 1 & $11.1 \%$ \\
\cline { 2 - 4 } & $\begin{array}{l}\text { Right ureterovesical } \\
\text { junction obstruction }\end{array}$ & 1 & $11.1 \%$ \\
\hline \multirow{4}{*}{$\begin{array}{l}\text { Renal } \\
\text { unit }\end{array}$} & Lt kidney & 8 & $44.4 \%$ \\
\cline { 2 - 4 } & Rt kidney & 8 & $44.4 \%$ \\
\cline { 2 - 4 } & upper moiety & 1 & $5.6 \%$ \\
\cline { 2 - 4 } & lower moiety & 1 & $5.6 \%$ \\
\hline
\end{tabular}

\section{Anatomical parameters measured by MRU} and Ultrasound:

Table (2) represents both AP diameter and parenchymal thickness measured by MRU and Ultrasound.

MRU AP diameter mean was $13.35 \pm$ $10.73 \mathrm{~mm}$ ranging from 4 to $42 \mathrm{~mm}$ whereas in Ultrasound AP diameter mean was $22.7 \pm$
$10.53 \mathrm{~mm}$ (which is higher than that of MRU)ranging from 6 to $34 \mathrm{~mm}$.

MRU parenchymal thickness mean was $8.14 \pm 3.02 \mathrm{~mm}$ ranging from 4 to $13 \mathrm{~mm}$ as opposed to ultrasound parenchymal thickness mean was $7.94 \pm 3.61 \mathrm{~mm}$ ranging from 3 to $18 \mathrm{~mm}$.

Table (2): Comparison between MRU and US as regard anatomical parameters.

\begin{tabular}{|l|c|c|}
\hline & $\begin{array}{c}\text { Mean } \pm \\
\text { SD(mm) }\end{array}$ & Range(mm) \\
\hline MRU AP diameter & $\begin{array}{c}13.35 \pm \\
10.73\end{array}$ & $4-42$ \\
\hline $\begin{array}{l}\text { MRU parenchymal } \\
\text { thickness }\end{array}$ & $\begin{array}{c}8.14 \pm \\
3.02\end{array}$ & $4-13$ \\
\hline $\begin{array}{l}\text { Ultrasound AP } \\
\text { diameter }\end{array}$ & $\begin{array}{c}22.73 \pm \\
10.53\end{array}$ & $6-34$ \\
\hline $\begin{array}{l}\text { Ultrasound } \\
\text { parencymal } \\
\text { thickness }\end{array}$ & $\begin{array}{c}7.94 \pm \\
3.61\end{array}$ & $3-18$ \\
\hline
\end{tabular}

\section{Detection of ureteric dilation in MRU and Ultrasound:}

8 of the patients that were shown to have ureteric dilation by ultrasound when repeated by MRU only 5 of them were shown to have ureteric dilation as shown in table 3 .

Table (3): Comparison between MRU and US as regard ureteric dilatation

\begin{tabular}{|l|c|c|c|c|}
\hline \multirow{2}{*}{} & \multicolumn{4}{|c|}{ Ureteric dilation } \\
\cline { 2 - 5 } & No dilatation & \multicolumn{2}{c|}{ Dilatation } \\
\cline { 2 - 5 } & $\mathbf{N}$ & $\mathbf{\%}$ & $\mathbf{N}$ & $\mathbf{\%}$ \\
\hline MRU & 13 & $72.2 \%$ & 5 & $27.8 \%$ \\
\hline Ultrasound & 9 & $52.9 \%$ & 8 & $47.1 \%$ \\
\hline
\end{tabular}

Non significant agreement (70\%) between MRU and Ultrasound in detection of ureteric dilatation with $\mathrm{p}$ value 0.07 proving that MRU can show us better delineation of the ureter as shown in table 4 
Table (4): Agreement between US and MRU as regard ureteric dilation

\begin{tabular}{|c|c|c|c|c|c|c|c|c|}
\hline & \multicolumn{2}{|c|}{ MRU ureteric dilation } & \multirow{2}{*}{ Total } & \multicolumn{4}{|c|}{ Agreement } \\
\hline & & No & Yes & & $\%$ & Kappa & p value & Sig. \\
\hline \multirow{2}{*}{$\begin{array}{l}\text { Ultrasound } \\
\text { ureteric dilation }\end{array}$} & no & $8(47.06 \%)$ & $1(5.88 \%)$ & $9(52.94 \%)$ & \multirow{3}{*}{$70.6 \%$} & \multirow{3}{*}{0.397} & \multirow{3}{*}{0.079} & \multirow{3}{*}{ NS } \\
\hline & yes & $4(23.53 \%)$ & $4(23.53 \%)$ & $8(47.06 \%)$ & & & & \\
\hline \multicolumn{2}{|l|}{ Total } & $12(70.59 \%)$ & $5(29.41 \%)$ & $17(100 \%)$ & & & & \\
\hline
\end{tabular}

\section{Urinary tract obstruction detection:}

Table (5) shows urinary tract obstruction detection in combined dynamic and static MRU in comparison to the basic imaging investigations.

Urinary tract obstruction was divided into 3 categories (normal, equivocal and obstructed). In combined MRU (both static and dynamic) $61.1 \%$ of cases was considered normal in comparison to $55.6 \%$ in the basic imaging protocol. $27.8 \%$ of cases was

Table (5): Comparison between MRU and other investigations as regard detection of urinary tract obstruction

\begin{tabular}{|l|c|c|c|c|c|c|}
\hline \multirow{2}{*}{ Urinary tract obstruction } & \multicolumn{2}{|c|}{ Normal (not obstructed) } & \multicolumn{2}{c|}{ Equivocal } & \multicolumn{2}{c|}{ obstructed } \\
\cline { 2 - 7 } & $\mathbf{N}$ & $\mathbf{\%}$ & $\mathbf{N}$ & $\mathbf{\%}$ & $\mathbf{N}$ & $\mathbf{\%}$ \\
\hline Dynamic and static MRU & 11 & $61.1 \%$ & 5 & $27.8 \%$ & 2 & $11.1 \%$ \\
\hline Basic imaging protocol & 10 & $55.6 \%$ & 5 & $27.8 \%$ & 3 & $16.7 \%$ \\
\hline
\end{tabular}

There was a significant agreement between combined static and dynamic MRU and other imaging investigations in detection of urinary obstruction with $\mathrm{p}$ value $<0.001$.

Table (6)Agreement between MRU and other investigations as regard urinary tract obstruction detection.

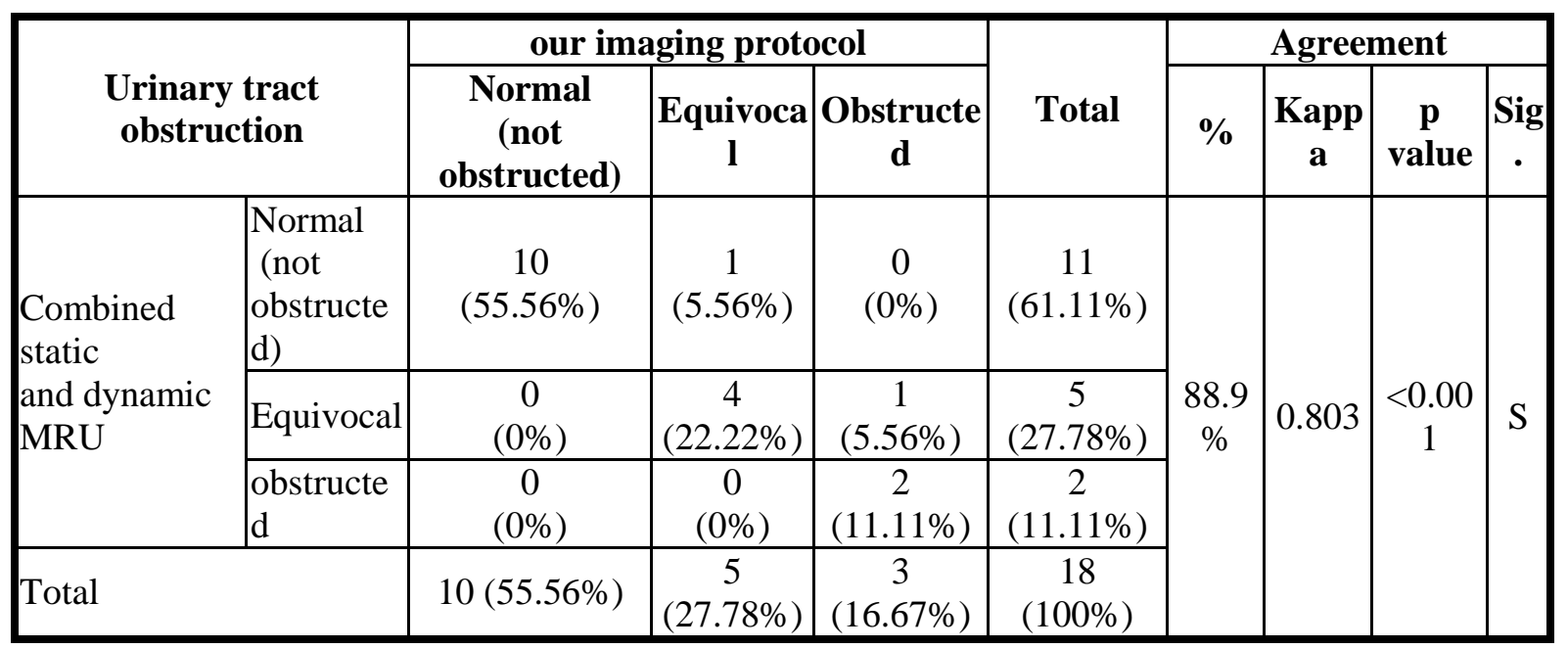

Table 7 shows that Renal transit time measured by dynamic MRU had poor agreement in our cases with urinary tract obstruction detected by other imaging protocol with a percentage of $46.2 \%$. 
Table (7): Comparison between RTT and other imaging as regard urinary tract obstruction detection.

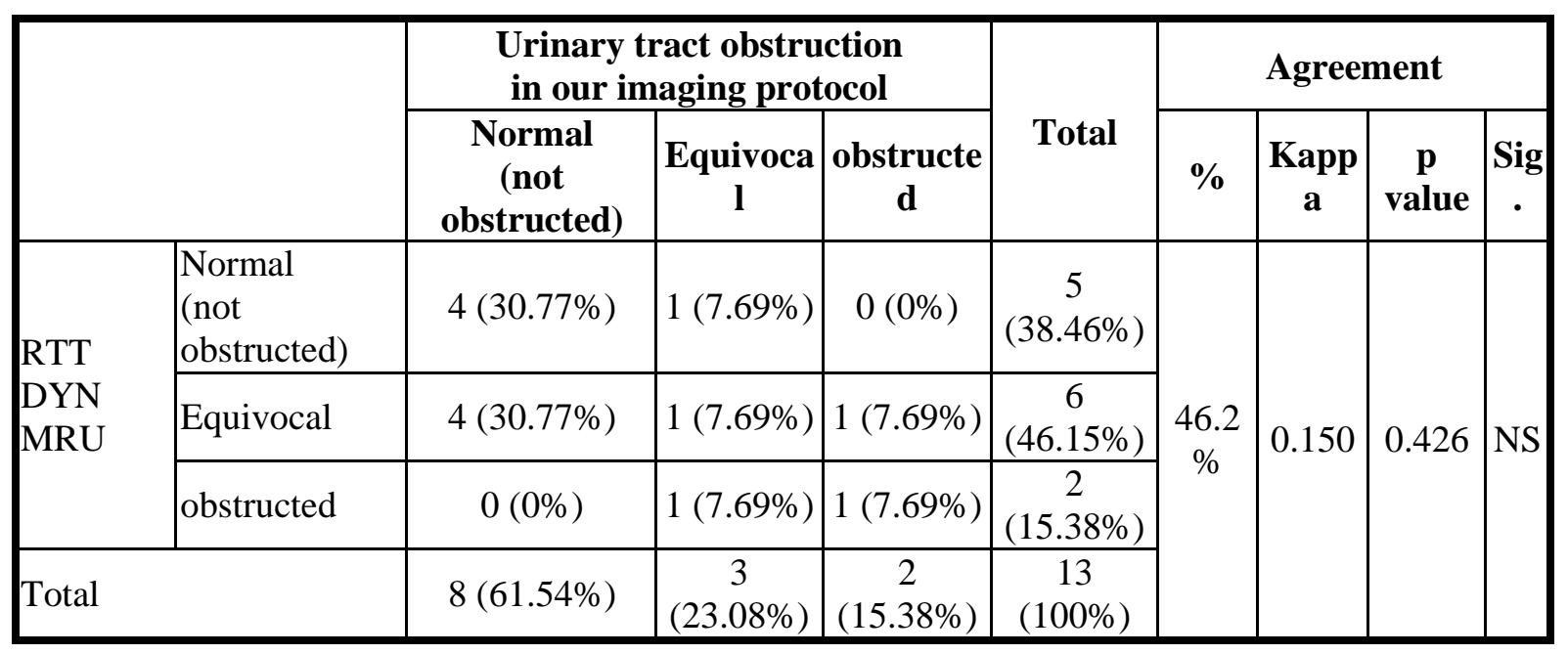

\section{Detection of obstruction level:}

Obstruction level was detected in $100 \%$ of the cases using the combined static and dynamic MRU in contrast to only $55.6 \%$ was shown by the other imaging protocol.

Table (8): Comparison between MRU and other imaging as regard detection of level of obstruction.

\begin{tabular}{|c|c|c|c|c|}
\hline \multirow{2}{*}{ Obstruction level } & \multicolumn{2}{|c|}{$\begin{array}{c}\text { Not } \\
\text { detecte } \\
\text { d }\end{array}$} & \multicolumn{2}{|c|}{ Detected } \\
\cline { 2 - 5 } & $\mathbf{N}$ & $\mathbf{\%}$ & $\mathbf{~ N}$ & $\%$ \\
\hline $\begin{array}{l}\text { combined static- } \\
\text { dynamic MRU }\end{array}$ & 0 & $\begin{array}{c}0.0 \\
\%\end{array}$ & 9 & $\begin{array}{c}100.0 \\
\%\end{array}$ \\
\hline other imaging protocol & 4 & $\begin{array}{c}44.4 \\
\%\end{array}$ & 5 & $\begin{array}{c}55.6 \\
\%\end{array}$ \\
\hline
\end{tabular}

Lin's Concordance correlation between MRU AP diameter \& Ultrasound AP diameter:

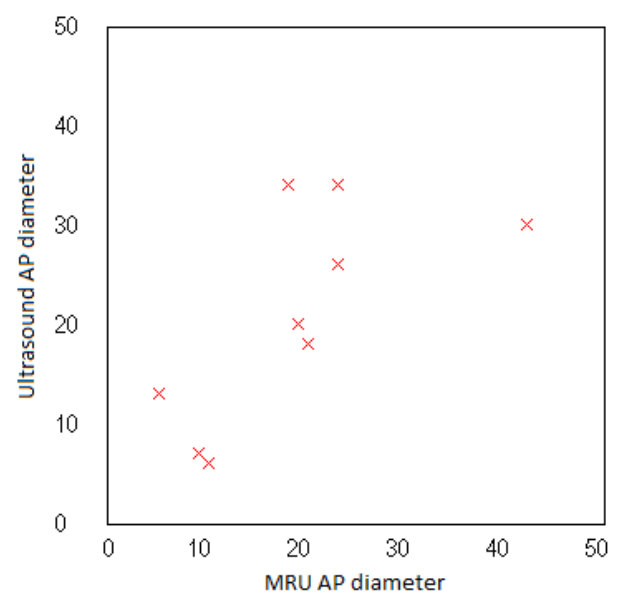

\begin{tabular}{|l|c|}
\hline $\begin{array}{l}\text { Lin's Concordance correlation } \\
\text { Coefficient pc }\end{array}$ & 0.677 \\
\hline $95 \%$ CI & $0.096-0.914$ \\
\hline
\end{tabular}

Fig. (1): Lin's Concordance correlation between MRU AP diameter \& Ultrasound AP diameter.

There was a poor agreement between MRU AP diameter measured with MRU and that of measured with Ultrasound with correlation coefficient 0.677 .

Lin's Concordance correlation between MRU parenchymal thickness \& Ultrasound parencymal thickness:

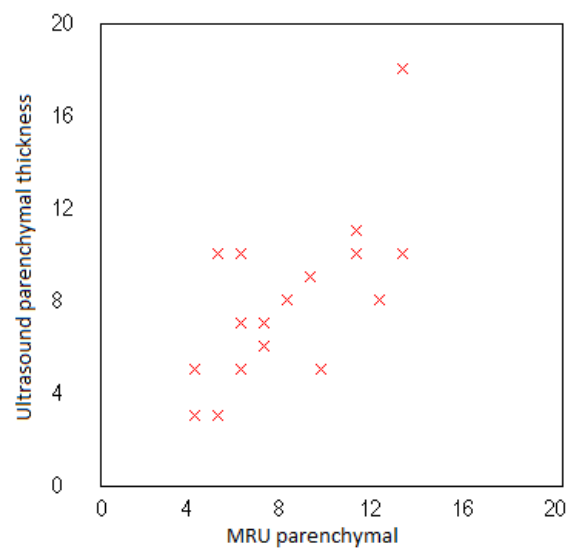




\begin{tabular}{|l|c|}
\hline $\begin{array}{l}\text { Lin's Concordance correlation } \\
\text { Coefficient } \rho c\end{array}$ & 0.663 \\
\hline $95 \% \mathrm{CI}$ & \\
\hline
\end{tabular}

Fig. (2): Lin's Concordance correlation between MRU parenchymal thickness \& Ultrasound parencymal thickness.

There was a poor agreement between MRU and Ultrasound in measuring the parenchymal thickness with Concordance correlation coefficient 0.663 .

\section{DISCUSSION}

Urinary tract obstruction is defined as any condition that impairs urinary drainage from the pelvicalyceal system and leads to increased pressure and reduced urine flow rate. This was the first approach to define obstructive uropathy with a more functional understanding ${ }^{(2)}$.

Clinically, obstruction is usually presumed when hydronephrosis is detected by ultrasound. But not every patient with hydronephrosis has an obstruction, and most cases of antenatal hydronephrosis will improve without surgical intervention or renal damage. It is important to state that hydronephrosis per se does not correlate with the affected kidney's function. It is still a challenge for physicians to identify whether the dilated system is obstructed or not ${ }^{(7)}$.

In children, impairment of renal drainage can limit the functional efficacy of the developing kidney. Ultrasound is the primary imaging investigation in dilatative uropathy complemented by voiding cystourethrography and less often by intravenous pyelography ${ }^{(8)}$.

Quantification of split renal function and possible obstruction is important for therapeutic decisions in children. For the assessment of split renal function and excretion, radionuclide scintigraphy is performed and can include the measurement of the plasma clearance of radioactive tracers technetium-99 m (99 $\mathrm{mTc})$ diethylenetriamine pentacetic-acid(DTPA), or 99 mTcmercapto-acetyl-triglycene (MAG3) ${ }^{(9)}$.

MR urography is an optimal imaging technique to evaluate morphologic abnormalities of the urinary tract without radiation exposure. MR imaging using gadolinium-chelates (Gd-DTPA) has been proposed for the evaluation of renal function and excretion ${ }^{(11,10)}$.

In our study we tried to explore the role of combined static and dynamic MRU in decision making in the management of pediatric upper urinary tract dilatation for the comprehensive assessment of renal morphology, excretion and split renal function by comparing the data obtained from MRU with our basic investigations.

\section{As regard the Dynamic MRU Functional results;}

Static and dynamic MR urography showed the morphology of the urinary tract and excretion with sufficient diagnostic imaging quality, and the results were in diagnostic compliance with scintigraphy.

This was in accordance with PerezBrayfield et al. ${ }^{(12)}$ in their study of 96 pediatric patients concluded that MR urography (MRU) accurately defined anatomical details and differential renal function in a single study without ionizing radiation. The calculation of relative renal function by MRU revealed excellent correlation with renal scintigraphy.

Also this was similar to Rohrschneider et al. ${ }^{(13)}$ who studied 62 patients of hydronephrosis using MRI and concluded that image quality was good or excellent in $95 \%$ of the cases and for split renal function, Dynamic MRU and isotope study showed significant correlation $(\mathrm{r}=0.92$, $\mathrm{p}<0.001$ ).

This study extends our technique of MR urography by adding the calculation of RTT and applying this calculation to the classification of obstructive uropathy in children. The technique can be performed by using clinical MR imagers, and the values for RTT, can be used in conjunction with highquality morphologic images of the urinary tract obtained in the same study. The combination of an objective time criterion, the SRF, and morphologic imaging in a single imaging session is a powerful indicator for obstruction and provides a useful tool for follow-up studies. 
In our study renal transit time showed poor agreement with detection of urinary obstruction in comparison to other imaging protocol with $46.2 \%$.

This contrasts with the previous finding by Jones et al. ${ }^{(14)}$ who calculated the renal transit time, and defined it as the time between the arrival of contrast material in cortex and its arrival in ureter, and found that it correlated well with the half- life or washout time of the contrast material used in renal scintigraphy studies showing good agreement with area under the curve 0.9 .

Also this contrasts with McDaniel et $a^{a}{ }^{(15)}$, in their study of the utility of dynamic MRU in PUJO in children, who noted that anatomic evaluation combined with renal transit time classification provided a reliable parameter for the identification of the obstruction.

We can explain this finding that In the presence of hydroureteronephrosis, there is only a gradual increase in signal, which makes the calculation of the RTT somewhat more subjective and in patients with megaureter, the compliance of the megaureter can lead to observance of a normal RTT (16).

Because of the excellent spatial and contrast resolution of contrast-enhanced MR urography, identification of excreted contrast medium in the ureters is straightforward. We chose to categorize the excretion patterns as non-obstructed, equivocal, and obstructed to correlate with typical categories described at renal scintigraphy.

\section{As regard the obtained anatomical results;}

In comparison to the basic investigations, the higher spatial resolution of the MR examination provides additional important information improving the management of the pediatric patients.

Combined static and dynamic MRU showed superiority to Ultrasound in identification and delineation of the anatomy with poor agreement between MRU and Ultrasound as regard the results of AP diameter, Parenchymal thickness and detection of ureteric dilation,that is due to subjective assessment of ultrasound depending on the operating personnel giving the MRU superior anatomical delineation of the renal morphology.

This was in accordance with PerezBrayfield et al. ${ }^{(12)}$ in their study of 96 pediatric patients concluded that MR urography (MRU) accurately defined anatomical details and differential renal function in a single study without ionizing radiation.

\section{As regard detection of obstruction level;}

Obstruction level was detected in $100 \%$ of the cases using the combined static and dynamic MRU in contrast to only $55.6 \%$ was shown by the other imaging protocol.

This was in accordance with Leppert et al. ${ }^{(17)}$ in their study on 24 hydronephrotic patients with surgery who compared preoperative data (USG, IVU, voiding cystourethrography, isotope renogram and MRU) with intraoperative findings and found that comparison of different imaging modalities proved MRU to be more accurate in the accurate localization of stenosis along the urinary tract and the morphology of renal parenchyma showing MRU the highest accuracy among all imaging modalities, with potential to replace IVU in the preoperative diagnostic workup of childhood hydronephrosis.

In contrast to this study we couldn't confirm the exact location of site of obstruction with intraoperative finding except only in the case of iatrogenic ureteric stricture.

The diversity of information given by MRU promises to make it an attractive method for assessment of obstructive uropathy.

Additionally, three dimensional images depicted anatomical anomalies very well in all patients.

Further studies can be done to evaluate the degree of hydronephrosis and to correlate it with the RTTs and to observe the hydronephrosis decreasing in those patients whom the RTTs improve.

Also we can study to what degree the dynamic mru study time may be reduced without a loss of diagnostic information, it 
should be addressed in a study with a larger number of patients to be evaluated.

Our study was limited by the small number of patients, due to scarcity of the dye in our country and only a limited amount could be imported. In addition to unavailability of anaesthesia at times and some technical obstacles regarding the body coil till another one could be obtained.However, the results of this methodological pilot study are very promising and further studies are justified.

Another limitation was that the proposed protocol needed a relatively long measuring time within the scanner of approximately 1 hour, which posed two problems: high health-care costs and a need for anaesthesia in the majority of the pediatric patients.

On the other hand, the MR nephrography protocol offers comprehensive information on morphology, excretion, and function, which otherwise could only be obtained by a multi-modal approach. Furthermore, standard procedures such as renal scintigraphy need a comparable amount of examination time, when a furosemide study is applied. Nevertheless, the assessment of excretion and split renal function by magnetic resonance imaging as an alternative to scintigraphy provides higher spatial resolution without application of radioactive nuclides.

After this pilot study, it is too early for the definition of quantitative borders indicating surgical treatment. At this moment of the research, the use of a qualitative evaluation of the obstruction by curve characteristics (prior and after the administration of furosemide) in addition to a relevant decrease of the split renal function and anatomical data and degree of dilatation seem best established and independent parameters in the decision making between a conventional or a surgical treatment, when symptoms and clinical course are doubtful.

\section{CONCLUSION}

Our results provide evidence that combined static and dynamic MRU is a promising technique that allows anatomical and functional evaluation of obstructed kidneys. It has a high sensitivity for detecting the level and cause of obstruction. MRU can be used as a single modality for the comprehensive evaluation of urinary tract obstruction.

\section{REFERENCES}

1. Rodriguez MM (2014): Congenital anomalies of the kidney and the urinary tract (CAKUT), Fetal and Pediatric Pathology, 33(5-6): 293-320.

2. Riccabona $M$ and Fotter $R$ (2008) Obstructive uropathy in childhood. In: Baert AL, editor. Encyclopedia of diagnostic imaging. Berlin: SpringerVerlag.

3. Peters CA (1995): Urinary tract obstruction in children. J Urol., 154(4):1874-1883.

4. Anumba DO, Scott JE, Plant ND et al. (2005): Diagnosis and outcome of fetal lower urinary tract obstruction in the northern region of England. PrenatDiagn., 25:7-13.

5. Gordon I, Piepsz A, Sixt R (2011): Auspices of Paediatric Committee of European Association of Nuclear M. Guidelines for standard and diuretic renogram in children. Eur $\mathrm{J}$ Nucl Med Mol Imaging, 38(6):1175-1188.

6. Servaes $S$ and Epelman $M$ (2013): The current state of imaging pediatric genitourinary anomalies and abnormalities. Curr Prob Diagn Radiol., 42(1):1-12.

7. Bianchi D, Vespasiani G, Bove P (2014): Acute kidney injury due to bilateral ureteral obstruction in children. World $\mathrm{J}$ Nephrol., 3(4):182-192.

8. Dias CS, Silva JM, Pereira AK et al. (2013): Diagnostic accuracy of renal pelvic dilatation for detecting surgically managed ureteropelvic junction obstruction. J Urol., 190(1):661-666.

9. Lee WG, Kim JH, Kim JM et al. (2010): Renal uptakes of 99mTc-MAG3, 99mTcDTPA, and 99mTcDMSA in rabbits with 
unilateral ureteral obstruction. In Vivo, 24:137-139.

10. Boss A, Martirosian $P$, Fuchs $J$ et al. (2014): Dynamic MR urography in children with uropathic disease with a combined $2 \mathrm{D}$ and $3 \mathrm{D}$ acquisition protocol-comparison with MAG3 scintigraphy. $\mathrm{Br}$ J Radiol., 87(1044):404-426.

11. Jones RA, Grattan-Smith JD and Little $S$ (2011): Pediatric magnetic resonance urography. J Magn Reson Imaging, 33(3):510-526

12. Perez-Brayfield MR, Kirsch AJ, Jones RA et al. (2003): A prospective study comparing ultrasound, nuclear scintigraphy and dynamic contrast enhanced magnetic resonance imaging in the evaluation of hydronephrosis. J Urol., 170:1330-1334.

13. Rohrschneider WK, Haufe S, Clorius JH et al. (2003): MR to assess renal function in children. Eur Radiol., 13:1033-1045.
14. Jones RA, Perez-Brayfield MR, Kirsch AJ et al. (2004): Renal transit time with MR urography in children. Radiology, 233:41-50.

15. McDaniel BB, Jones RA, Scherz $H$ et al. (2005): Dynamic contrastenhanced MR urography in the evaluation of pediatric hydronephrosis. Part 2. Anatomic and functional assessment of uteropelvic junction obstruction. AJR., 185(6):16081614.

16. Chevalier RL and Peters CA (2003): Congenital urinary tract obstruction: proceedings of the State-Of-The-Art Strategic Planning Workshop-National Institutes of Health, Bethesda, Maryland USA, 11-12 March 2002. Pediatr Nephrol., 18:576-606.

17. Leppert A, Nadalin S, Schirg E et al. (2002): Impact of magnetic resonance urography on preoperative diagnostic workup in children affected by hydronephrosis: should IVU be replaced? J Pediatr Surg., 37:1441-1445. 